\title{
1 Title: Environmental sensing in dynamic quorum responses
}

3 Authors: Eric K. Chu ${ }^{1,2,3}$, Alex Groisman ${ }^{4}$ and Andre Levchenko ${ }^{1,2,3, *}$

4 Affiliations (at the time the work was performed):

5 1. Department of Biomedical Engineering, Yale University, New Haven, Connecticut 06520,

$6 \quad$ USA

7 2. Yale Systems Biology Institute, Yale University, West Haven, Connecticut 06516, USA

8 3. Department of Biomedical Engineering, The Johns Hopkins University School of

9 Medicine, Baltimore, Maryland 21205, USA

10 4. Department of Physics, University of California, San Diego, La Jolla, California 92093,

$11 \quad$ USA

12 * Please address the correspondence to andre.levchenko@yale.edu 


\section{Abstract}

15 Cell communication and coordinated cell behavior are hallmarks of multicellular behavior of

16 living systems. However, in many cases, including the ancient and archetypal example of

17 bacterial quorum sensing, the meaning of the communicated information remains a subject of

18 debate. It is commonly assumed that quorum sensing encodes the information on the current

19 state of the colony, including cell density and physical colony confinement. Here, we show that

20 quorum sensing can also be exquisitely sensitive to dynamic changes in the environment,

21 including fluctuations of the prevailing nutrient source. We propose a new signaling mechanism

22 accounting for this sensory capability. This mechanism combines regulation by the commonly

23 studied lux operon-encoded network with the environmentally determined balance of protein

24 synthesis and dilution rates, dependent on the rate of cell proliferation. This regulatory

25 mechanism accounts for observed complex spatial distribution of quorum responses, and

26 emergence of sophisticated processing of dynamic inputs, including temporal thresholds and

27 persistent partial induction following a transient change in the environmental conditions. We

28 further show that, in this context, cell communication in quorum sensing acquires a new

29 meaning: education of cells within a population about the past history of transient exposure to

30 adverse conditions by a subset of induced cells. In combination, these signaling and

31 communication features may endow a cell population with increased fitness in diverse

32 fluctuating environments. 


\section{$34 \quad$ Introduction}

35 Collective cell behaviors coordinated by inter-cellular communication are ubiquitous across all

36 domains of life ${ }^{1-5}$, supporting evolutionary advantages of multicellular vs. cell-autonomous

37 signal detection and responses. However, the information conveyed in cell-cell communication

38 and its consequences for the population fitness in dynamically changing environments are

39 frequently not known. Quorum sensing (QS) is an ancient archetypal cell-cell communication

40 mechanism found in many prokaryotes ${ }^{6}$. It is used to coordinate collective cell responses,

41 through endogenously-produced diffusible molecules called autoinducers $(\mathrm{AI})^{7,8}$. What exactly is

42 sensed in the process of QS, however, is a matter of debate. Classically, QS has been defined as a

43 means to condition adaptive responses on achieving sufficient cell density (CD), which is gauged

44 by the accumulation of AI with increasing cell number ${ }^{9,10}$. However, AI can also accumulate to

45 high levels due to poor diffusive characteristics of the local environment, even if the cell density

46 is relatively low ${ }^{11,12}$. This observation led to an alternative interpretation of AI-mediated QS

47 signaling as an active method of surveying the diffusive (DF) transport characteristics of the

48 extracellular milieu ${ }^{13}$. Importantly, QS is frequently coupled to stress-response phenotypes such

49 as sporulation $^{14,15}$, virulence ${ }^{16-18}$, and biofilm formation ${ }^{19-21}$, indicating that at least some aspects

50 of QS can report on adverse environmental conditions. Indeed, poor local diffusive properties or

51 high cell density might also hamper nutrient availability ${ }^{22}$; however, neither CD nor DF sensing

52 interpretations directly relate to the detection of changes in the environmental conditions,

53 particularly of subtle fluctuations in nutrient content. It is not clear, therefore, whether QS might

54 in fact be a mechanism to sense dynamic changes in the potentially stressful, dynamic cell

55 microenvironment ${ }^{23}$, or it just enables this sensing (e.g., in a cell density-dependent fashion)

56 through alternative sensory mechanisms ${ }^{24}$. 
58 To explore the function of QS, it is important to investigate the dynamic features of QS

59 responses. However, the emphasis has traditionally been placed on the categorical on or off

60 description, stemming from the common feature of the molecular circuits underlying QS - the

61 positive feedback. Positive autoregulation is observed, for example, in a frequently studied QS

62 circuit of the marine bacterium $V$. fischeri, whose response is controlled by the lux operon ${ }^{25}$. It

63 involves AI, produced by the AI synthase LuxI, binding to its cognate cytoplasmic AI receptor

64 LuxR, and positively auto-regulating the $L u x I$ and $L u x R$ in addition to driving bioluminescence

65 gene transcription ${ }^{26-28}$. This simple feedback regulatory circuit enables a switch-like increase in

66 the QS response after the threshold concentration of AI is exceeded ${ }^{29}$. However, the time scale of

67 this response and its single-cell properties remain poorly understood. It is therefore not clear how

68 the dynamics of QS may compare to other key time scales, including the duration of cell cycle,

69 environmental fluctuations or, for example, in the case of $V$. fischeri, the day-night life cycle of

70 the host (i.e., the Hawaiian Bobtail Squid, Euprymna scolopes).

72 Here, using a highly integrated combination of experimental and computational approaches,

73 relying in particular on a new method to tightly control the coupling between bacterial

74 populations and variable environmental conditions, we found several unexpected properties of

75 QS. In particular, we found that QS cannot be fully accounted for by the current CD and DF

76 sensing theories, thus requiring a new framework for its interpretation. We propose such a

77 framework and provide extensive quantitative evidence for its validity. Furthermore, we find that

78 dynamic, rather than static, QS response can be a critical determinant of the corresponding

79 phenotypic outcomes. In addition, our results suggest that QS can display complex patterns of 
80 spatial distribution and variability, allowing for an increased fitness in the uncertain

81 environments, through a communication-driven cell 'education' mechanism. These findings can

82 have important consequences for our understanding of QS and the related phenotypic outcomes,

83 and can have implications for other QS-like phenomena, including eukaryotic community effects

84 and other cell communication-related behaviors.

$\underline{\text { Results }}$

87 QS is defined by a balance between nutrient-defined protein synthesis and dilution due to

\section{8 cell proliferation}

89 To explore whether QS is consistent with either the CD or DF interpretation (Fig. 1a), we

90 studied the QS responses of densely packed colonies of E. coli lab strain MG02S ${ }^{30}$ exposed to

91 various tightly-controlled environments within a microfluidic device. MG02S carries a single,

92 genomically integrated copy of $V$. fisheri LuxIR QS circuit regulating GFP expression in lieu of

93 bioluminescence genes (Fig. 1b). The cells were grown to a completely dense monolayer state

94 within 720 microchambers of identical dimensions, but with variable numbers and sizes of

95 connective channels which modulate the diffusive coupling between the microchambers and the

96 medium-supplying flow-through channels (Fig. 1c, e, Fig. S1a, b). We found that, despite

97 achieving dense colonies of $\sim 4000$ cells each that were grown chemostatically for 24 hours, cells

98 exhibited no detectable QS in the presence of glucose-rich $(20 \mathrm{mM})$ tryptone-complemented

99 medium (TCM) at $25^{\circ} \mathrm{C}$ (Fig. S2a, Movie 1). However, switching to glucose-deficient TCM

100 elicited a strong auto-induced QS response, consistent with previous reports of glucose-mediated

101 repression of the $l u x$ operon $^{31,32}$. Furthermore, despite the same cell density and number within

102 each chamber, the magnitude of QS responses across chambers varied for different coupling 
103 configurations (Fig. 1d, S3). Thus, the QS responses was not fully consistent with the CD

104 interpretation, but might instead have reflected DF sensing, in which QS response is defined by

105 the diffusion-limited local accumulation of AI. We indeed confirmed that different

106 environmental coupling of individual chambers resulted in variable transport (Fig. S1c, d, Movie

107 2, 3) and cell growth rates (Fig. 1e, Fig. S1e-g, Movie 4). The transport and cell growth were

108 closely related to each other (Fig. S1h), suggesting that diffusive coupling controlled both AI

109 accumulation and cell proliferation via nutrient access. However, contrary to what was expected

110 from the DF sensing interpretation, the magnitude of QS induction (at 24 hours following the

111 medium switch) was not a monotonic decreasing function, but rather exhibited a biphasic

112 dependence on cell growth rate (and diffusive coupling), (Fig. 1f, i, Movie 5), with the response

113 maximized at intermediate growth rates.

115 Further, to directly test whether differential AI accumulation between chambers was responsible

116 for the different QS responses, as would be predicted by the DF sensing hypothesis, we repeated

117 the experiment in the presence of $1 \mu \mathrm{M}$ of exogenous $\mathrm{AI}$, which is expected to saturate the

118 circuit and thus result in uniform QS induction across different chambers. Unexpectedly,

119 induction with exogenous AI again resulted in a biphasic QS induction pattern, essentially

120 identical to that resulting from auto-induction, albeit with different kinetics (Fig. 1i, Fig. S2b,

121 Movie 6), suggesting that neither CD nor DF sensing hypothesis was sufficient to explain the

122 observed responses.

123

124 Since QS response varied with cell growth rate, we tested whether it would change with another

125 source of nutrients. Strikingly, replacing tryptone with a very similar nutrient source, casamino 
126 acids (tryptone results from partial and casamino acids from complete digestions of casein,

127 respectively), resulted in a very distinct, more homogeneous response distribution, albeit still

128 biphasic in shape, for both auto- and exogenous AI induction conditions (Fig. 1i). Even more

129 surprising, given the similarity of the nutrients, was the dramatically different dynamics of the

130 QS response in the presence of TCM vs. casamino acid complemented medium (CACM) (Fig.

131 1g, Fig. S2c, S4a, b, Movie 7-9).

132

133 Our results suggest that neither CD nor DF interpretation is completely adequate to account for

134 the observed QS responses, with the growth rate being a more predictive determinant of the

135 response than either cell density or local accumulation of AI. An explanation of this growth

136 dependency might be provided by the dynamics of QS response, and in particular, the

137 observation that the time scales of QS are comparable to and can exceed the duration of the cell

138 cycle. Therefore, the dynamics and amplitude of the response can be strongly influenced not only

139 by the biochemical interactions within the molecular QS circuit, as is commonly assumed, but

140 also by the dynamic balance between protein synthesis and growth-mediated protein dilution

141 (Fig. 1h). We indeed found that the synthesis and dilution (which can also be converted to

142 protein retention, as shown here) of QS-regulated protein (GFP) were monotonically increasing

143 functions of the cell growth rate (Fig. 1j) for both tryptone and casamino acids as nutrient

144 sources. However, the average growth rate was higher and synthesis rate was lower for the

145 tryptone vs. casamino acids complemented media. To test whether the balance of QS protein

146 synthesis and dilution would be able to account for various experimentally observed response

147 dynamics, we modified previously published mathematical models of QS response ${ }^{33,34}$ to

148 incorporate the measured synthesis and dilution parameters from the different conditions (Fig. 
S5). By matching the synthesis and dilution rate constants to the experimentally estimated

150 values, we obtained the predicted QS dynamics that was consistent with the experimental

151 observations for both nutrient sources (Fig. 1f, g, Fig. S6). This result provided first evidence for

152 the key influence of the balance between protein synthesis and dilution as a regulatory

153 mechanism of the environmental sensitivity of the QS response.

Information about distinct environmental conditions is reflected in QS response dynamics

156 To further support the protein synthesis/dilution balance (PSD) mechanism, we explored QS

157 under additional environmental perturbations. In particular, we reasoned that increasing the

158 temperature would accelerate cell growth and thus increase protein dilution, leading to an

159 attenuated QS response. Indeed, increasing the temperature from $25^{\circ} \mathrm{C}$ to $30^{\circ} \mathrm{C}$ elevated cellular

160 growth rates resulting in a complete abrogation of response in TCM even after 48 hours of

161 observation (Fig. 2a,b, Fig. S2d, Movie 10). The same temperature increase in CACM, which

162 has reduced growth rates, resulted in a partial QS onset (88 out of 144 chambers assayed) that

163 was delayed by approximately 15-20 hours, matching the mathematical model predictions (Fig.

164 S2e, Movie 11). To directly test whether protein abundance could modulate the onset of the QS

165 response, we transformed the cells with a construct coding for IPTG-inducible LuxR expression

166 (Fig. S4c), and used it to examine the effect of LuxR overexpression on QS induced in TCM at

$16730^{\circ} \mathrm{C}$. Overexpression of LuxR resulted in the rescue and immediate onset of QS response in all

168 chambers even at the elevated temperature (Fig. 2c, Fig. S2f, Movie 12). As expected, the timing

169 of the onset of signaling was also dramatically shortened with overexpression of LuxR in TCM

170 at $25^{\circ} \mathrm{C}$, with responses in all chambers triggered virtually immediately (Fig. S2g, Movie 13).

171 Alternatively, if the level of glucose used for cell culture prior to the switch to TCM was lowered 
172 (20 to $10 \mathrm{mM}$ ), which was expected to increase the basal level of LuxR expression due to both

173 partial catabolic de-repression and reduced cell growth, a decrease in the QS onset timing and

174 increase in the amplitude was observed (Fig. S2h, Movie 14). Although these experiments

175 suggested the importance of achieving a high level of LuxR expression for QS onset, the onset

176 time was also dramatically decreased if exogenous AI was added at the time of the QS induction

177 in TCM and CACM at $25^{\circ} \mathrm{C}$ (Fig. S2b, c). This result implied that QS onset can be accelerated if

178 the abundance of either LuxR or LuxI (and thus AI) is increased in the cells, which can be

179 controlled by the balance of synthesis and dilution of these proteins, as dictated by growth

180 conditions. On the other hand, consistent with the results above, the response magnitude, unlike

181 the response dynamics, was insensitive to exogenous AI (Fig. 1i, Fig. S4d), implying that AI,

182 under these conditions primarily controls the dynamics of the QS responses (Fig 2d). All of these

183 results were also well approximated by the corresponding versions of the mathematical model,

184 further supporting the PSD mechanism (Fig. S2, 6).

186 Diverse conditions used in the experiments suggested that the QS outcome can be encoded not 187 only in the magnitude but also in the timing of the onset and the initial rate of the QS response

188 (Fig. 2a, d, Fig. S6). This dynamic information can inform downstream responses long before

189 the steady state QS response is achieved. It is thus of interest to explore the sensitivity of the

190 dynamic aspects of QS to the various environmental conditions and to the degree of coupling

191 between the colony and the environment. One way to assay the sensitivity to environmental

192 inputs is an information theoretic approach ${ }^{35,36}$, which, given the response variability, can

193 indicate how many input types or doses can be accurately resolved. Using this approach, we

194 found that the onset time showed the highest sensitivity in discriminating external environmental 
195 conditions explored here, yielding $1.76 \pm 0.03$ bits of information, equivalent to accurately

196 distinguishing $2^{1.76}=3.4$, or approximately 3 different types of conditions (Fig. 2d, e, Fig. S7).

197 This discrimination capacity was in spite of the variability of environmental coupling across the

198 chambers, suggesting that the timing of the QS onset can provide the early information about the

199 changes in the environment regardless of the exact nature of the coupling of environment to the

200 cellular niche. Conversely, the response magnitude was the metric that was most sensitive to the

201 differential diffusive coupling, with at least 1 bit of information representing the ability to

202 accurately distinguish at least 2 coupling levels for each environmental condition (Fig. 2d, f, Fig.

203 S8). The onset timing, on the other hand, was the least informative about this aspect of the cell

204 environment, consistent with the results above. This result suggested that the degree of cellular

205 confinement in a particular niche can indeed be conveyed by QS, but information about this

206 aspect of cell environment does not become available until high levels of response are attained.

208 We noted that our calculation of the information about the environmental coupling was likely an

209 underestimation, since the biphasic nature of the QS response distributions meant that two

210 distinct mean colony growth rates could correspond to the same mean QS response magnitude,

211 creating an ambiguity that can reduce the information content in the QS response magnitude.

212 Therefore, relatively slow and relatively fast growth can potentially result in the same QS

213 response magnitude and trigger the same levels of downstream adaptive response, unless there is

214 an additional means to resolve this ambiguity, a possibility that we explored next.

215

216 Spatial properties of environmentally regulated QS response 
217 We hypothesized that the ambiguity associated with the biphasic dependence of the mean QS

218 response on the degree of coupling (growth rate) could potentially be resolved, if two different

219 chambers yielding the same average QS response magnitude at different growth rates would

220 have distinct spatial distributions of the QS signal. In particular, consumption of the nutrients

221 diffusing into the chambers by multiple cells within the chambers can lead to gradients of

222 nutrient availability, and thus, corresponding gradients of the cellular growth rates. As a result,

223 each chamber can span a range of growth rates, with growth rates higher in the chamber regions

224 adjacent to the coupling channels due to proximity to the nutrient source. We thus hypothesized

225 that the range of growth rates within a chamber can result in the range of QS responses

226 corresponding to the biphasic distribution curve measured before (Fig. 3a). Consequently, the

227 spatial gradients of QS response within a chamber would correspond to a segment of the biphasic

228 dependence curve, with the local inclination of the segment specifying the direction of the spatial

229 gradient (Fig. 3b). The biphasic nature of the curve would, therefore, specify gradients of

230 opposite signs, according to the rising and falling parts of the biphasic curve. Since the two

231 values of growth rates corresponding to the same QS response can map to two parts of the

232 biphasic curve with the opposite slopes, the resulting gradients of QS responses would be

233 opposite, providing extra information that can resolve the ambiguity discussed above.

235 We indeed found that different chambers incubated in TCM at $25^{\circ} \mathrm{C}$ for 24 hours had distinct

236 spatial QS magnitude distributions, and that the spatial gradient directions were fully consistent

237 with the hypothesis above, and thus the PSD hypothesis (Fig. 3b-d Fig. S9a). We further

238 explored if this hypothesis could also account for the dynamics of spatial QS response evolution.

239 In particular, we investigated if this spatial dynamics can be accounted for by the dynamics of 
240 the mean QS responses (Fig. S10, 11). For example, the QS response distribution is biphasic

241 throughout the 24 hours of QS induction in TCM at $25^{\circ} \mathrm{C}$, implying that the location of

242 maximum QS response in chambers with lower average growth rates would be proximal to the

243 nutrient-supplying channels throughout the response, starting from onset (Fig. 3c-e, Fig. S10,

244 11). On the other hand, the maximal response would occur in more distal regions for chambers

245 with higher average growth rates. Indeed, we found that the onset position for QS in both TCM

246 and CACM conditions corresponded to the location of the maximal response as well as the local

247 inclination of the biphasic curve, shifting from proximal to distal regions of the chamber when

248 examining chambers with increasing mean growth rates (Fig. 3f, Fig. S9b, S12). Overall, these

249 results suggest that both the average QS response magnitude and the spatial gradients of the QS

250 response in individual chambers can be accounted for by the same mechanism. This observation

251 suggests that the biphasic nature of the response magnitude distribution, explained by the PSD

252 mechanism, can lead to a variety of spatial QS distributions, as a function of coupling between

253 the cellular niches and extracellular milieu.

\section{Temporal thresholds and bistability in QS response}

256 Our data suggests that the dynamics of QS response can convey the information on a persistent

257 change in the environmental conditions, particularly the nutrient content, with this information

258 further refined as a function of the degree of diffusive coupling, resulting in nonuniform spatial

259 distributions of the signaling magnitude. However, the changes in the environment are frequently

260 transient or display complex dynamics. We thus investigated how QS responds to such dynamic

261 conditions. In particular, we investigated if variable QS onset timing can translate into condition-

262 dependent temporal thresholding, requiring a persistent rather transient change in the 
263 environment, longer than a certain threshold, for the response to occur (Fig. 4a). We explored

264 this possibility by transiently switching from the glucose-rich TCM to the QS-inducing TCM at

$26525^{\circ} \mathrm{C}$ for 4 hours, which is shorter than the fastest onset timing of $\sim 7$ hours in these conditions

266 (Fig. 1h). This transient change in the environment indeed resulted in no detectable QS response

267 in both simulations and experiments, suggesting a temporal filtering of inputs below a threshold

268 duration (Fig. 4b, Fig. S13a, Movie 15). Furthermore, periodic changes in cell environment

269 between TCM and glucose-rich medium (4 hour-long pulses, 50\% duty cycle) again resulted in

270 the absence of QS response, implying no temporal integration of the transient inputs (Fig. S13b,

271 Movie 16). On the other hand, a 16-hour stimulation with the TCM, exceeding the slowest QS

272 onset time among cells, enabled the response in all chambers (Fig. 4b, Fig. S13c, Movie 17).

273 This response recovered to the baseline in all but 3 chambers within 24 hours after restoring

274 glucose-rich conditions. In combination, these results suggested effective temporal filtering of

275 the environmental changes by the QS circuit, ensuring that only sufficiently persistent signals

276 activate the response.

278 The 3 chambers retaining the QS signaling following transient stimulation were the ones with the

279 slowest growth rates (Fig. 4b, Fig. S14a). Since transient responses can be stabilized by the

280 positive feedback and the associated bistability and hysteresis, we hypothesized that the QS-

281 associated positive feedback might be maintaining the response in a subset of chambers, even in

282 the presence of the glucose-rich environmental conditions. Further analysis and our prior work ${ }^{30}$

283 suggest that the bistability regime, and thus, the number of chambers retaining the response

284 following transient stimulation, can be expanded by lowering glucose concentrations in the

285 medium prior to and following the transient stimulation (Fig. 4c). Indeed, for cells pre-incubated 
in $10 \mathrm{mM}$ glucose (mean QS onset time of $7.5 \pm 1.5$ hours), glucose removal for 4 hours was

287 sufficient to elicit QS in several, but not all, chambers, again supporting the existence of a

288 temporal threshold, albeit lowered for this condition (Fig. 4b, Fig. S13d, 14b, Movie 18).

289 Consistent with the previous results, the chambers showing the QS response in this experiment

290 were also the ones with slower growth rates and thus included those that displayed persistent

291 response after cessation of the stimulus. As hypothesized, the number of chambers where QS

292 was persistently retained after removal of the stimulus increased to 7 . Extending the stimulus

293 duration to 9 hours permitted all chambers to become transiently induced and achieve higher

294 amplitudes of induction, but did not affect the number of chambers displaying irreversible QS

295 (Fig. S13e, Movie 19). These results highlighted a strong bi-stable nature of the response

296 consistent with the positive feedback interactions within the lux signaling circuit ${ }^{27,30}$ leading to a

297 'memory' of a prior induction even if the environmental conditions no longer favor QS. Overall,

298 we concluded that better environmental coupling and more nutritious environment can impart

299 longer temporal thresholds ensuring a low and transient QS response for a subset of chambers

300 exposed to a transient change in the environment (Fig. 4c). On the other hand, poorer

301 environmental coupling and less nutritious environments can each reduce the temporal threshold

302 and ensure persistent QS responses in an increasing number of micro-chambers (Fig. 4c).

Cell communication allows a small pre-induced cell sub-population to quicken the onset of

305 response in the rest of the population through cell communication

306 Visual inspection of chambers with persistent QS response revealed that only small fractions of

307 cells were highly induced, with the rest of the population displaying no detectible QS signaling

308 (Fig. S14, Movie 17-19). The higher-than-basal levels of AI produced by the induced 
309 subpopulation could be key to the maintenance of the high stable state of expression of QS

310 genes $^{37,38}$ in the presence of active environmental suppression. Indeed, simultaneous induction

311 and repression with exogenous $\mathrm{AI}$ and glucose, respectively, stably maintained an induced

312 subpopulation (Fig. S15, Movie 20). Furthermore, we hypothesized that this subset of cells could

313 carry a memory of a prior stimulation, which can provide a selective advantage if conditions

314 become adverse, and thus, stimulatory again. Although this strategy is superficially similar to the

315 commonly assumed 'hedging of bets' scenario ${ }^{39,40}$, which postulates that a diversification of

316 response within a population can confer a selective advantage under uncertain environmental

317 conditions, the cell-cell communication nature of QS imparts additional benefits. In particular,

318 the induced cells can potentially promote induction of neighboring cells after worsening of

319 conditions through secretion of AI, thus quickening the timing of the QS onset in the uninduced

320 cells, permitting a greater fraction of cells to faster assume the required adaptive phenotype. We

321 thus explored if cell communication inherent to QS can indeed reduce the temporal threshold and

322 accelerate the onset of response.

324 To compare the temporal threshold between fully uninduced and partially induced cell

325 populations, we modified the microfluidic platform to screen distinct environmental conditions

326 that can yield such populations simultaneously. More specifically, we explored 12 chamber

327 configurations in the presence of several glucose concentrations in the 0-6 $\mathrm{mM}$ range (Fig. 4d,

328 Fig. S16), which enabled us to elicit a wide spectrum of responses ranging from complete

329 presence to complete absence of induction within the chamber configuration with the highest

330 diffusive coupling (Fig. S17a, Movie 21). We found that in the 0-0.86 mM range of glucose

331 concentrations, the cells in the colonies were fully induced, whereas in the 1.71-6 mM range, we 
332 observed bi-stable responses, with chambers showing different fractions of induced cells that

333 were stably maintained for at least 40 hours after initial onset (Fig. 4e, g, Movie 21). In the cases

334 of 1.71 and $2.57 \mathrm{mM}$, the bi-stability resulted in either complete (ranging from $18.77 \pm 8.26 \%$ to

$33597.26 \pm 0.72 \%$ ) or partial (ranging from $0.037 \pm 0.066 \%$ to $12.19 \pm 6.82 \%$ ) induction in different

336 chambers, while chambers in the 4.29-6 $\mathrm{mM}$ range contained either partially induced or

337 completely un-induced (ranging from $0.0015 \pm 0.0009 \%$ to $0.0079 \pm 0.0047 \%$ ) cell populations

338 (Fig. S18). This range of distinct bi-stable responses allowed us to examine whether and how the

339 presence of different fractions of induced cells would affect the QS response (Fig. 4f).

341 To accomplish this, we studied the effect of switching to TCM from all glucose supplemented

342 conditions, which resulted in a complete QS induction for all chambers, but with distinct onset

343 times (Fig. 4h, Fig. S17b, Movie 22, 23). In all cases, we compared, for the same glucose level,

344 the onset timing of uninduced cells either in the fully uninduced populations or populations

345 containing a fraction of induced cells, due to the underlying bi-stability. For all glucose levels,

346 we found that the presence of a fraction of induced cells dramatically shortened the QS onset

347 time in uninduced cells (Fig. 4i, Fig. S19). Notably, the most dramatic relative change in the

348 onset timing was observed between 3.43 and $4.29 \mathrm{mM}$ of glucose in the pre-incubation medium,

349 also coinciding with the lowest glucose levels allowing bi-stable responses and thus presence of

350 induced sub-populations. This result was consistent with AI-mediated cell-to-cell communication

351 from the induced subset of cells, pre-conditioning the remaining uninduced cells in the

352 population and thus promoting a quicker QS induction following the onset of adverse conditions.

353 This was also consistent with the faster onset of QS in separate experiments whereby cells were

354 exposed to low doses of exogenous AI (Fig. S20, Movie 24-26). These experiments suggested 
355 that if the fitness of the population is coupled to the QS onset, the presence of the persistently

356 induced sub-population can provide a selective advantage to the whole population by enabling a

357 faster response to a dynamic change in the colony environment.

359 Discussion

360 QS is one of the most ancient mechanisms of cell communication, and yet, its functional role

361 remains ambiguous. It has been proposed that, in addition to the widely accepted cell density

362 sensing function, QS can also serve to assess the diffusive transport properties of the

363 microenvironment. Our results provide a more complex view of this archetypal signaling

364 mechanism, demonstrating that QS signaling is also sensitive to dynamic changes of

365 environmental conditions such as nutrient composition and access. The observed QS responses

366 are best explained not only by the currently accepted view of QS as driven by positive feedbacks

367 inherent in most QS genetic circuits, including the lux operon explored here, but also by the

368 balance of protein synthesis and dilution modulated by the specific microenvironments that the

369 cells are exposed to. In combination, these regulatory mechanisms endow the QS response with

370 several new properties that can strongly affect the fitness of the cell population and the outcome

371 of the adaptive processes frequently coupled to the QS activation.

373 We found that a subtle change in the nutrient source, such as the degree of digestion of casein,

374 can strongly influence the balance of the rates of cell growth rate and protein synthesis,

375 translating into the dilution and synthesis of the QS-mediating gene products. In particular, a

376 higher degree of digestion translated into a substantial increase in protein synthesis rate

377 accompanied by a decrease in cell proliferation. The reciprocal balance between protein 
378 synthesis and cell proliferation may reflect a more general strategy of allocation of limited

379 available resources to different intracellular processes ${ }^{41,42}$. Importantly, this balance can also be

380 influenced by inputs other than nutrients, including, as shown here, gene amplification or altered

381 temperature. The resulting complex regulation translates into non-monotonic responses of the

382 long term QS amplitude as a function of colony growth rate, which in turn translates into the

383 distinct distributions of the spatial QS responses, with the gradients of the response pointing

384 either toward or away from the nutrient source. This inherent non-monotonicity might also

385 explain complex differentiation patterns of B. subtilis into a state of competence ${ }^{43}$, suggesting

386 similar regulation in a distinct underlying QS circuit.

388 Successful adaption may depend not only on mounting the responses that are adequate to the

389 environmental challenges, but also on how quickly these responses can be mounted. The

390 bistability inherent in the positive feedback regulation of most known genetic QS circuits, when

391 modulated by the environmentally defined variable balance between growth rate and protein

392 synthesis, allows for complex decision-making. In particular, this regulatory mechanism can

393 ignore pulsatile environmental inputs, such as changes in nutrient content, that are too transient,

394 while responding to more persistent stimuli by either transient or persistent activation, lasting

395 long past the input cessation. The balance between protein synthesis and dilution is crucial for

396 establishing the time it takes to reach the critical concentrations of the regulatory proteins

397 enabling the QS response, and thus for controlling the temporal thresholding of inputs and the

398 timing of response initiation. This sensitivity of the initial response dynamics to the temporal

399 fluctuations of the extracellular milieu can supply the information on environmental changes to 
400 the downstream, adaptive circuits far in advance of reaching the steady state of the QS response,

401 which may provide additional time for eliciting appropriate responses.

402

403 Similar considerations pertain to the cases of QS involvement in symbiotic settings, as for $V$.

404 fischeri, where the circadian cycles of animal foraging are synchronized with cycles of growth

405 and QS response by the symbiont bacterial cells. We find that amino acid-based nutrient sources,

406 thought to be the means of the host's support of $V$. fischeri growth in vivo, may support QS

407 response only in a certain range of temperatures, likely present in the context of ocean animals,

408 and with highly adjustable onset time, allowing the response to be regulated by nutrients not only

409 through control of cell density, but also more directly.

411 Our results also reveal a new aspect of the possible meaning of the messages exchanged by cells

412 during QS-related cell-communication. The persistent responses of cell sub-populations

413 following transient changes in the cell environment can serve as a type of memory of the recent

414 exposure to conditions promoting QS, even if the environment has since become benign. This

415 memory is carried not by the population as a whole but by different fractions of cells within the

416 population, which can be quite limited. As mentioned above, this strategy of diversification of

417 QS responses within a population may resemble the often discussed bet-hedging response, in

418 which a sub-population of cells may be more adapted to a possible change in the environment

419 than the rest of the population. However, in the case of the QS response, the non-autonomous

420 nature imparted by cell-cell coupling through secreted AI can allow the induced subpopulation,

421 which can anticipate the environmental change, to accelerate response by the uninduced cells, if

422 the conditions indeed change for the worse. This allows the uninduced cells to avoid the 
423 metabolic costs associated with the initial QS induction but still enable faster onset of QS-

424 associated adaptive response when needed. In a sense, this represents a type of 'education' of

425 uninduced cells by the induced ones, providing the intercellular communication messages with a

426 specific meaning. The resulting higher overall population responsiveness can increase the

427 population fitness vs. the populations devoid of such cell 'education' capabilities.

$429 \underline{\text { Methods }}$

430 Design and fabrication of microfluidic device

431 The overall organization of the channels and chambers in the microfluidic device is similar to

432 that in previous studies ${ }^{44,45}$. Briefly, an array of 16 parallel flow-through channels with a depth of

$43315 \mu \mathrm{m}$ are connected to 24 rows of 30 chambers each, which house the cells. The dimensions of

434 the chambers are $100 \times 100 \times 0.8 \mu \mathrm{m}$, with a $15 \times 15 \mu \mathrm{m}$ post in the center. The degree to which

435 the chambers are connected to the flow-through channels via coupling channels are varied. 16

436 rows of chambers are connected to flow-through channels by coupling channels only on one

437 single side of the chamber, while eight rows of chambers are connected to flow-through channels

438 by coupling channels on two, opposing sides of the chamber. Each side of the chamber that is

439 connected to the flow-through channels has either one, two, or three coupling channels, giving

440 one-sided chambers a total of one, two, or three coupling channels, while double-sided chambers

441 have a total of two, four, or six coupling channels. In addition, the coupling channel dimensions

442 are 25 or $50 \mu \mathrm{m}$ in length, and 10 or $20 \mu \mathrm{m}$ in width. The combination of different dimensions

443 and configurations of coupling channels produced 24 unique chamber types with varying degrees

444 of mass transport properties. The chambers are organized into four quadrants of six types each,

445 with three single-sided and three double-sided chambers in each quadrant. Each group is then 
446 distributed in a tandem, repeated triplet fashion, to ensure that each chamber type is distributed

447 evenly throughout the quadrant to minimize the effect of positional dependence relative to the

448 source of medium.

450 Growth medium is supplied from either one of two inlets, which connect to the flow-through

451 channels and supply nutrients to chambers on its way to a single outlet port, while the fluid from

452 the alternate inlet is directed to a waste port. Because the height of the chambers are relatively

453 shallow compared to the height of the flow-through channels, the chambers are much more

454 resistant to flow across the chamber as compared to flow through the channels, hence, the

455 dominant mode of mass transport into the chamber is through diffusion. The symmetric binary

456 branching of the flow-through channels ensures that the pressures are balanced between

457 channels, further preventing crossflow into the chamber. Flow is driven through the device via

458 hydrostatic pressure differences, with the height of the syringe connected to the inlet with the

459 desired medium higher than the alternate inlet, which are both higher than the syringes connected

460 to the outlet and waste port. The medium being supplied to the chambers from one inlet can be

461 instantaneously switched to that of the alternate inlet by swapping the height of the syringes,

462 allowing for rapid changes in the medium conditions.

464 A chemical gradient-generating microfluidic device was also used in this study, based off of the

465 Christmas tree design ${ }^{46}$. Similar to the device mentioned above, the gradient device contains 2

466 inlets, 1 outlet, and 1 waste port, allowing for similar operation under normal conditions.

467 However, between the chambers and the inlets are a series of serpentine channels, which can

468 perform mixing of solutions if medium from both inlets are supplied simultaneously. If one inlet 
469 (source) contains a molecule which the other inlet (sink) lacks, each stage of serpentine channels

470 performs progressively more refined dilutions, resulting an increasingly resolved concentration

471 gradient spanning the two concentrations of the inlets. Individual concentration doses are

472 separated into channels, allowing downstream chambers to be exposed to different

473 concentrations. The device creates 8 linearly graded concentration doses from 2 input

474 concentrations, each connected to a row of 30 (only double-sided) or 90 (single- and double-

475 sided) chambers, depending on the design. The design with 30 chambers in each row has only a

476 chamber type with the highest degree of connectivity, while the design with 90 chambers in each

477 row has 12 chamber types, all of which are contain $25 \mu \mathrm{m}$ long coupling channels. The heights

478 of the chamber and channels are the same as the device above, except the design with 90

479 chambers in each row have chamber heights of $\sim 0.75 \mu \mathrm{m}$. The channels converge into the outlet

480 via the same symmetric binary branching structure, allowing for equal pressures. When a

481 gradient is desired, it can be generated by equating the pressure from both syringes and

482 supplying medium from both inlets at the same time. A fluorescent dye is used to visualize the

483 gradient.

485 The device was fabricated in a similar fashion as in previous studies ${ }^{44,45}$. Briefly, the initial

486 design was drawn in Adobe Illustrator and sent to be printed as a photomask. The photomask

487 was used to produce a master mold via photolithography. The master mold was made with a 3"

488 silicon wafer with a two-level micro-relief $(0.8 \mu \mathrm{m}$ and $15 \mu \mathrm{m})$ of a UV-curable epoxy (SU-8 by

489 MicroChem, Newton, MA). The first level was made with SU-8 2002 spun onto the silicon wafer

490 at an initial $500 \mathrm{rpm}$ for 10 seconds with an acceleration index of 1 , then $10000 \mathrm{rpm}$ for 30

491 seconds with an acceleration index of 100 , to produce a $\sim 0.8 \mu \mathrm{m}$ thick film. The chambers were 
492 patterned with photolithography, followed by subsequent baking and development to form the

493 structures. The subsequent level was made with SU-8 2015 spun onto the silicon wafer at an

494 initial $500 \mathrm{rpm}$ for 10 seconds with an acceleration index of 1, then $3250 \mathrm{rpm}$ for 30 seconds

495 with an acceleration index of 2 to produce a $\sim 15 \mu \mathrm{m}$ thick film. The channels were patterned and

496 made as above. Microfluidic devices were then fabricated with PDMS (Sylgard 184, Dow

497 Corning) via soft lithography. A $5 \mathrm{~mm}$ thick cast of PDMS made with 10:1 ratio of elastomer

498 base to elastomer curing agent. The PDMS cast was peeled off the wafer and cut into individual

499 chips, and ports were punched with a 20 gauge luer stub. Devices were washed and hermetically

500 sealed to \#1.5 microscope cover slips, then baked in a $130^{\circ} \mathrm{C}$ oven overnight prior to use.

\section{Strain and growth conditions}

503 MG1655 E. coli expressing a truncated Lux quorum sensing operon from $V$. fischeri made in a 504 previous study ${ }^{30}$ was used. Briefly, MG01S, containing luxR divergently transcribed from PluxI

505 fused to GFP, and MG02S, which is identical to MG01S bar the addition of LuxI upstream of

506 GFP and under the same promoter, were made by the restriction digests of EcoRI-KpnI and

507 EcoRI-BamHI fragments of the lux operon from pLVA01 and pLVA02, respectively, and cloned

508 into pPROBE'-GFP-Tagless. An EcoRI-NotI digestion of the resulting plasmid and subsequent

509 cloning into $\lambda \mathrm{InCh}$ vector allowed for the genomic integration of the lux quorum sensing circuit

510 into the E. coli MG1655 chromosome. $100 \mathrm{ug} / \mathrm{mL}$ ampicillin was added for selection.

512 The inducible LuxR strain was made by PCR amplification of the LuxR gene from MG02S with 513 forward primer $5^{\prime}-$

514 ATCTCTGAATTCCCGTTTTAATGATATATAACACGCAAAACTTGCGAC - 3', which 
515 adds an EcoRI restriction site upstream of the LuxR, and reverse primer 5' -

516 CAAGTATGGTACCCGTACTTAACTTTTAAAGTATGGGCAATCAATTGCTCC - 3’,

517 which adds a KpnI restriction site downstream of the gene. The PCR fragment was cloned into

518 pEXT22 plasmid by digesting both with EcoRI and KpnI, placing LuxR under the control of a tac

519 promoter. The subsequent plasmid was transformed into MG02S. LuxR overexpression was

520 induced with $1 \mathrm{mM}$ IPTG added to growth medium. $50 \mathrm{ug} / \mathrm{mL}$ kanamycin was added for

521 selection.

523 Prior to every experiment, a single colony was selected from a plate and inoculated into LB with

524 the appropriate antibiotics and grown overnight at $30^{\circ} \mathrm{C}$ with shaking at $230 \mathrm{rpm}$. The next day,

525 the overnight culture was diluted 1:100 into CACM (2\% casamino acids, 1x M9 salts (12.8g

$526 \mathrm{Na}_{2} \mathrm{HPO}_{4} 7 \mathrm{H}_{2} \mathrm{O}, 3 \mathrm{~g} \mathrm{KH} \mathrm{KHO}_{4}, 0.5 \mathrm{~g} \mathrm{NaCl}$ and $1 \mathrm{~g} \mathrm{NH} 4 \mathrm{Cl}$ per liter), $1 \mathrm{mM} \mathrm{MgCl}$ ) with $20 \mathrm{mM}$

527 Succinate, $20 \mathrm{mM}$ Glucose and $100 \mathrm{ug} / \mathrm{mL}$ ampicillin, and grown at $30^{\circ} \mathrm{C}$ with shaking at 230

528 RPM until the culture reached an $\mathrm{OD}_{600}$ of $0.2-0.3$ (approximately 3-4 hours). The culture was

529 subsequently centrifuged and cell pellet re-suspended in 1\% BSA in PBS prior to loading into

530 the device. Cell loading was performed from the outlet port of a BSA in PBS-primed device.

531 After loading into the device, growth medium with the appropriate antibiotics and $20 \mathrm{mM}$

532 glucose is supplied to the cells continuously via one or two inlets and the cells are grown at $30^{\circ} \mathrm{C}$

533 until all chambers are full before beginning any experiment, unless otherwise indicated. Other

534 growth media used were all variations of the CACM, with $20 \mathrm{mM}, 10 \mathrm{mM}$, or absence of

535 glucose, or the substitution of $2 \%$ casamino acids with $2 \%$ tryptone, both of which are sourced

536 from digestions of casein. 


\section{Microscopy}

539 Widefield image acquisition was performed on a Nikon Eclipse TE2000 epifluorescence inverted

540 microscope equipped with a motorized stage (Prior Scientific, Cambridge, UK) and a Cascade

541 1K EMCCD camera (Photometrics, Tucson, Arizona). Timelapse images were acquired with a

542 Nikon Plan Fluor 40x/0.75 Ph2 DLL objective, while montage images were captured with a

543 Nikon Plan Fluor 10x/0.3 Ph1 DL objective, and stitched together automatically with 10\% image

544 overlap with a custom MATLAB script. Both phase contrast and fluorescence images were

545 captured for timelapse and montage images. Timelapse images were acquired every 20 minutes,

546 with an exposure time of 100-500 ms, while the montage images were taken with an exposure

547 time of $1000 \mathrm{~ms}$. The excitation filter wavelengths used for GFP detection was 450-490nm

548 (Chroma, Rockingham, VT), while the emission filter wavelengths was 525nm. The excitation

549 filter wavelengths used for detection of Alexafluor 555 was $541-565 \mathrm{~nm}$, while the emission

550 filter wavelengths was $584-679 \mathrm{~nm}$. A spectral 2D template autofocus algorithm was used to

551 maintain the focus for the entire duration of timelapse imaging. For time-lapsed images, at least

552 two positions for each condition/chamber type was chosen for each experiment, while montages

553 varied from 42 to 77 images depending on the device used. Control of the microscope and

554 capture of images were performed in an automated fashion via Slidebook 5.5 (Intelligent

555 Imaging Innovations, Denver, Colorado).

557 Z-stacks were acquired with a Leica SP8 Laser Scanning Confocal microscope equipped with a

558 white light pulsed laser with continuous wavelength and hybrid detectors. Images were acquired

559 on a HC PL APO 40x/1.30 Oil, CS2 objective and the Leica Application Suite X software. 


\section{Evaluation of transport and growth in microfluidic device}

562 The transport properties of the various chamber types were evaluated with a dye, which served as

563 a proxy for a diffusible molecule and allowed for the visualization of the diffusion process.

564 Alexa Fluor 555 Hydrazide (Thermo Fisher Scientific, Waltham, MA) was chosen as the dye due

565 to its relatively high molecular weight as compared to most diffusible molecules of interest

$566(1150$, vs $213.23 \mathrm{~g} / \mathrm{mol}$ for AI and $180.16 \mathrm{~g} / \mathrm{mol}$ for glucose), giving it a smaller diffusion

567 coefficient and longer diffusion time. Thus, it served as a good representation of the dynamics of

568 even the slowest diffusing molecules while also accounting for the dynamics of a wide range of

569 molecules with faster diffusion dynamics. Cells were grown in dye-free medium within the

570 chambers overnight until the chambers were filled to capacity. $0.01 \mathrm{mM}$ of dye solution was

571 perfused into the flow-through channels by increasing the hydrostatic pressure of the dye-

572 containing medium syringe relative to the dye-free medium syringe. Fluorescence timelapse

573 microscopy was used to capture the fluorescence intensity within the chambers, at a rate of 1

574 minute per time point, for a duration of 15 minutes. The diffusion of the dye out of the chamber

575 was also monitored by switching back to the dye-free medium syringe. The intensity of the

576 fluorescence in the chamber is quantified by taking the mean of the intensity of the entire

577 chamber for each time point, and normalized to the initial or final timepoint, for diffusion out

578 and diffusion in, respectively. The transport properties of the various chamber types was also

579 modeled computationally with COMSOL Multiphysics 4.2 (COMSOL Inc. Stockholm,

580 Sweden), the results of which was in agreement with experiments.

582 The growth rate of the cells within the different chambers was assessed with the MG01S strain,

583 which lacks the ability to produce LuxI. Therefore, the production of high levels of GFP relies on 
584 the addition of exogenous AI, and ceases upon its removal. MG01S is loaded into chambers and

585 grown in the presence of $1 \mu \mathrm{M}$ AI to induce uniform GFP production until all chambers are filled

586 to capacity. Once AI is removed, fluorescence in the chamber is gradually lost as the continual

587 growth of the cells within the chamber would lead to the distribution of a fixed number of GFP

588 protein to the progeny, which are gradually pushed out of the chamber by the growing and

589 dividing cells within the chamber and carried away by the flow. Due to the stability of the GFP

590 used $^{47}$, the loss of GFP fluorescence is expected to be predominantly from dilution of the protein

591 as a result of growth and division of the cells, the rate of which would be proportional to the

592 growth rate within different chambers. The average fluorescence over time can be fitted with an

593 exponential curve of the form $\mathrm{a}^{*} \exp (\mathrm{bt})$, giving an estimate of the growth rate. The growth rate

594 was estimated for all 24 chamber types with both casamino acids and tryptone-based minimal

595 media, and at 25 and $30^{\circ} \mathrm{C}$.

\section{Image processing and data analysis}

598 Automated image acquisition was performed with Slidebook 5.5. Raw images were exported in

599 tiff format and processed using custom MATLAB scripts. All images were corrected for uneven

600 illumination with the following correction: $\mathrm{C}=(\mathrm{I}-\mathrm{D}) /(\mathrm{F}-\mathrm{D}) * \mathrm{M}$, where $\mathrm{C}$ is the corrected image, $\mathrm{I}$

601 is the initial image, $\mathrm{D}$ is the darkfield image, $\mathrm{F}$ is the flatfield image, and $\mathrm{M}$ is the mean of the

602 flatfield minus darkfield images. The flatfield and darkfield were averages of multiple images.

603 Time-lapse images were aligned automatically with an alignment algorithm, while montage

604 images were stitched together with the same alignment algorithm with $10 \%$ overlap between

605 adjacent images. Each calculated metric is an average of at least 3 independent experiments of at

606 least 2 replicates each for each chamber configuration, unless otherwise indicated. 
608 The automated data analysis pipeline imports the processed image file and determines the square

609 chamber region, excluding the post, containing cells. The mean intensity value for the entire

610 chamber was determined for both sets of image types. For time-lapse images, this is done for all

611 time points, and additional metrics were also calculated. To visualize the spatial distribution of

612 response along the $\mathrm{y}$-axis, the intensity values were averaged along the $\mathrm{x}$-axis and the resultant

613 column of intensity values were normalized by subtracting the minimum and dividing by the

614 minimum-subtracted maximum. The steepness of spatial gradients at each time point was

615 approximated with the slope from a linear regression performed on the column of intensity

616 values versus a normalized distance of 1, with a positive slope representative of higher responses

617 in the distal regions relative to the coupling channels, and a negative slope representative of

618 higher responses in the proximal regions relative to the coupling channels. Double-sided

619 chambers are analyzed as half-chambers along the y-axis due to symmetry, and the calculated

620 metric for both halves are averaged into a single value to represent the whole chamber.

621 Aggregating these intensity value columns for each time point generated the kymographs used

622 for spatial analysis. Onset time is defined as the time point at which more than $1.5 \%$ of the pixels

623 within the square region of the chamber have intensity values greater than mean $+4 * \mathrm{SD}$ of the

624 same region in the initial frame. Onset location was quantified from the kymographs. Briefly, the

625 columns of normalized intensity values from the onset time point to a time point 2 hours later

626 were extracted, and the positions with a normalized value of greater than or equal to 0.99 were

627 collected and the mean and standard deviations were calculated. The initial rate was estimated

628 with the slope from a linear regression on the first 5 hours of response after the onset time within

629 exogenous induction conditions, which is expected to reach maximal levels of production. Time- 
630 lapse fluorescence intensity measurements are normalized to the initial value. Two chamber

631 configurations were excluded (filled shapes) from the chip-wide measurements obtained from

632 the montage image analysis due to partial formation of cell bilayer, but z-sections from confocal

633 microscopy confirm the biphasic nature (Fig. S3). Fractions of induction within a chamber were

634 determined by the fraction of total pixels that exceeded a predetermined threshold value. For

635 categorization, mean $\pm \mathrm{SD} \leq 10 \%$ and $\geq 0.01 \%$ were considered partially induced, less than that is

636 uninduced, and greater than that is fully induced. For chambers that are not packed at the

637 beginning of the experiment, segmentation of cells based on phase contrast images were

638 performed, and all quantified data from these chambers were from the segmented images.

640 Z-stacks from confocal microscopy were split into individual focal planes, with the chamber

641 region for each plane segmented and the mean intensity calculated. The slice with the highest

642 mean intensity was used for analysis, and were compared to the mean value from the maximum

643 intensity projections.

\section{Computation of mutual information}

646 Briefly, mutual information $(I)$ between two random variables is calculated from the formula:

$647 \quad I(R ; S)=H(R)-H(R \mid S)$,

648 where $S$ and $R$ denote the signal and response, respectively, and $H$ is the entropy function:

$H(X)=-\sum_{i}^{n} p\left(x_{i}\right) \log _{2} p\left(x_{i}\right)$

Hence, formula (1) becomes: 


$$
\begin{aligned}
I(R ; S)= & \sum_{j}-P\left(R=r_{j}\right) \log _{2} P\left(R=r_{j}\right) \\
& -\sum_{i} P\left(S=s_{i}\right)\left(\sum_{j}-P\left(R=r_{j} \mid S=s_{i}\right) \log _{2} P\left(R=r_{j} \mid S=s_{i}\right)\right) .
\end{aligned}
$$

653 The marginal distribution of the response is

$654 P\left(R=r_{j}\right)=\sum_{i} P\left(S=s_{i}\right) P\left(R=r_{j} \mid S=s_{i}\right)$.

655 Since the formulas as shown above are for discrete data, the experimental data used for the

656 calculations, which lies on a continuous spectrum, are discretized by binning. However, binning

657 of the finite data sample results in biased estimates of the mutual information. Since bias is a

658 function of sample size, which approaches zero as sample size approaches infinity, we utilized

659 the series expansion of mutual information in terms of inverse sample size to estimate the

660 unbiased mutual information:

$661 \quad I_{\text {biased }}=I_{\infty}+\frac{a_{1}}{N}+\frac{a_{2}}{N^{2}}+\cdots$

662 Here, $I_{\text {biased }}$ is the biased estimate of mutual information, $I_{\infty}$ is the unbiased estimate of mutual

663 information with infinite sample size, $\mathrm{N}$ is the total number of samples, and $a_{i}$ are coefficients

664 dependent on the signal and response distributions. For sufficiently large N, all terms larger than

665 first order are negligible, resulting in the biased estimate of mutual information being a linear

666 function of inverse sample size. As a result, we can use jackknife sampling to sample subsets of

667 the data to compute the mutual information. By plotting these biased estimates of mutual

668 information to the inverse sample size, fitting a line, and extrapolating to infinite sample size, we 
can obtain the unbiased estimate of mutual information. For a more comprehensive description,

670 we refer the reader to previously published work ${ }^{35,36}$.

\section{Model and simulations}

673 Simulation of diffusion

674 Diffusion dynamics were simulated using COMSOL Multiphysics 4.2. Briefly, the 3D geometry

675 of each chamber configuration was recreated within the software, and the concentration of the

676 entire volume of the chamber was set to an initial concentration of $0 \mathrm{mM}$. All surfaces of the

677 chamber were set to have null flux except for the surfaces that interface with the flow-through

678 channels, which are set to a constant concentration of $0.01 \mathrm{mM}$, corresponding to the

679 concentration of dye used in the experiment. A diffusion coefficient of $2 \times 10^{-10} \mathrm{~m}^{2} \mathrm{~s}^{-1}$ was

680 used for the Alexa Fluor 555 dye. Time-dependent transport of diluted species simulations were

681 performed for all 24 chamber configurations. The average concentration within the chamber

682 volume was determined and plotted.

\section{Simulation of QS response}

685 The mathematical model is a simplified version of a model from a previous study ${ }^{34}$, accounting

686 for only one cell, but with new species and terms added:

$687 \frac{d[R]}{d t}=k_{6}+\frac{k_{3}[C]}{K_{D R}+[C]}-k_{10}[R]-k_{1}[R][A]+k_{-1}[R A]-k_{11}[R]$

$688 \frac{d[R A]}{d t}=k_{1}[R][A]-k_{-1}[R A]-2 k_{2}[R A]^{2}+2 k_{-2}[C]-k_{11}[R A]$

$689 \frac{d[C]}{d t}=k_{2}[R A]^{2}-k_{-2}[C]-k_{11}[C]$ 
$690 \quad \frac{d[I]}{d t}=k_{7}+\frac{k_{4}[C]}{K_{D I}+[C]}-k_{11}[I]-k_{8}[I]$

$691 \quad \frac{d[G]}{d t}=k_{7}+\frac{k_{4}[C]}{K_{D I}+[C]}-k_{11}[G]-k_{9}[G]$

$692 \frac{d[A]}{d t}=-k_{1}[R][A]+k_{-1}[R A]-k_{-13}[A]+k_{13}\left[A_{e}\right]+k_{5}[I]$

$693 \frac{d\left[A_{e}\right]}{d t}=k_{-13}[A]-k_{13}\left[A_{e}\right]-k_{12}\left[A_{e}\right]$

694 Diffusion of AI is only considered as a rate of loss from the cell, if no exogenous AI is

695 introduced. In addition, the production of AI from $\mathrm{C}$ has been explicitly separated into

696 production of LuxI from C (which then produces AI) to enable the account of LuxI dilution.

697 Because GFP is downstream of LuxI, the added equation is nearly identical, with a different 698 degradation term the only difference. Dilution terms were also added to the equations of all 699 protein species to account for loss of protein from cell division.

701 The global constants that are applicable to all the conditions are listed in Table 1, most of which

702 were based on a previous study ${ }^{33}$. For each environmental condition, a new set of $\mathrm{k}_{4}$ (synthesis,

703 Table 2) and $\mathrm{k}_{11}$ (dilution, Table 3) values were used, the range of which corresponds to the

704 different chamber configurations, and were measured from experiments. $\mathrm{k}_{4}$ (synthesis) was

705 estimated with the slope from a linear regression corresponding to the first 5 hours of response

706 after the onset time within exogenous induction conditions, which is expected to reach maximal

707 levels of production. Dilution rates are proportional to growth rate, so $\mathrm{k}_{11}$ (dilution) is calculated

708 in the same way with an exponential fit of the GFP dilution data. The exceptions are $\mathrm{k}_{4}$

709 (synthesis) for tryptone and casamino acids conditions at $30^{\circ} \mathrm{C}$, which were inferred by

710 comparing the change in growth rates and synthesis rates from tryptone to casamino acids 
711 medium at $25^{\circ} \mathrm{C}$, and extrapolating that relationship to the change in temperature from $25^{\circ} \mathrm{C}$ to

$71230^{\circ} \mathrm{C}$ in both media types.

713

714 Simulations were performed in MATLAB. Briefly, each simulation was run for an initial 24

715 simulation hours with either the LuxR repressed or LuxR partially repressed $\mathrm{k}_{3}$ and $\mathrm{k}_{6}$ values, to

716 replicate the initial growth in $20 \mathrm{mM}$ or $10 \mathrm{mM}$ glucose medium, respectively, and to allow for a

717 basal steady state amount of proteins and components to be made. The $\mathrm{k}_{3}$ and $\mathrm{k}_{6}$ values are

718 subsequently switched to the derepressed or overexpressed form, and simulation resumed for at

719 least another 24 simulation hours. Simulations corresponding to the transient glucose switching

720 experiments consisted of transient changes in the $\mathrm{k}_{3}$ and $\mathrm{k}_{6}$ values at the appropriate time points.

722 Statistical Analysis

723 At least 3 independent tests were performed for each experiment unless otherwise stated. F-test

724 was performed to determine variances between samples. 2-tailed t-tests were used for

725 comparisons.

726

727 Code Availability

728 The codes used in this study are available from the corresponding author upon request.

\section{$730 \quad$ Data Availability}

731 The data that support the findings of this study are available from the corresponding author upon

732 request.

733

734 
7351 Gestwicki, J. E. \& Kiessling, L. L. Inter-receptor communication through arrays of

736 bacterial chemoreceptors. Nature 415, 81-84, doi:10.1038/415081a (2002).

7372 Ajon, M. et al. UV-inducible DNA exchange in hyperthermophilic archaea mediated by

$738 \quad$ type IV pili. Mol Microbiol 82, 807-817, doi:10.1111/j.1365-2958.2011.07861.x (2011).

7393 McLean, B. G., Hempel, F. D. \& Zambryski, P. C. Plant intercellular communication via plasmodesmata. Plant Cell 9, 1043-1054, doi:10.1105/tpc.9.7.1043 (1997).

4 Puliafito, A. et al. Collective and single cell behavior in epithelial contact inhibition. Proc Natl Acad Sci U S A 109, 739-744, doi:10.1073/pnas.1007809109 (2012).

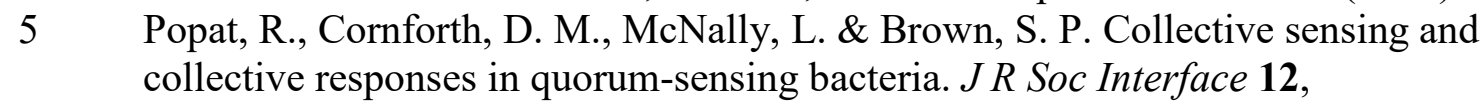
doi:10.1098/rsif.2014.0882 (2015).

6 Miller, M. B. \& Bassler, B. L. Quorum sensing in bacteria. Annu Rev Microbiol 55, 165199, doi:10.1146/annurev.micro.55.1.165 (2001).

7 Ng, W. L. \& Bassler, B. L. Bacterial quorum-sensing network architectures. Annu Rev Genet 43, 197-222, doi:10.1146/annurev-genet-102108-134304 (2009).

8 Shapiro, J. A. Thinking about bacterial populations as multicellular organisms. Annu Rev Microbiol 52, 81-104, doi:10.1146/annurev.micro.52.1.81 (1998).

9 Swift, S. et al. Quorum sensing as a population-density-dependent determinant of bacterial physiology. Adv Microb Physiol 45, 199-270 (2001).

10 Gao, M. et al. A crucial role for spatial distribution in bacterial quorum sensing. Sci Rep 6, 34695, doi:10.1038/srep34695 (2016).

12 Hense, B. A. et al. Does efficiency sensing unify diffusion and quorum sensing? Nat Rev

11 Boedicker, J. Q., Vincent, M. E. \& Ismagilov, R. F. Microfluidic confinement of single cells of bacteria in small volumes initiates high-density behavior of quorum sensing and growth and reveals its variability. Angew Chem Int Ed Engl 48, 5908-5911, doi:10.1002/anie.200901550 (2009). Microbiol 5, 230-239, doi:10.1038/nrmicro1600 (2007).

13 Redfield, R. J. Is quorum sensing a side effect of diffusion sensing? Trends Microbiol 10, 365-370 (2002).

14 Perchat, S. et al. How Quorum Sensing Connects Sporulation to Necrotrophism in Bacillus thuringiensis. PLoS Pathog 12, e1005779, doi:10.1371/journal.ppat.1005779 (2016).

15 Steiner, E., Scott, J., Minton, N. P. \& Winzer, K. An agr quorum sensing system that regulates granulose formation and sporulation in Clostridium acetobutylicum. Appl Environ Microbiol 78, 1113-1122, doi:10.1128/AEM.06376-11 (2012).

16 Antunes, L. C., Ferreira, R. B., Buckner, M. M. \& Finlay, B. B. Quorum sensing in bacterial virulence. Microbiology 156, 2271-2282, doi:10.1099/mic.0.038794-0 (2010).

17 Rumbaugh, K. P. et al. Kin selection, quorum sensing and virulence in pathogenic bacteria. Proc Biol Sci 279, 3584-3588, doi:10.1098/rspb.2012.0843 (2012).

18 Zheng, J., Shin, O. S., Cameron, D. E. \& Mekalanos, J. J. Quorum sensing and a global regulator TsrA control expression of type VI secretion and virulence in Vibrio cholerae. Proc Natl Acad Sci U S A 107, 21128-21133, doi:10.1073/pnas.1014998107 (2010). biofilm communities by surface-enhanced resonance Raman scattering. Nat Mater 15, 1203-1211, doi:10.1038/nmat4720 (2016). 
$78020 \quad$ Kim, M. K. et al. Surface-attached molecules control Staphylococcus aureus quorum sensing and biofilm development. Nat Microbiol 2, 17080, doi:10.1038/nmicrobiol.2017.80 (2017).

$78321 \quad$ Parsek, M. R. \& Greenberg, E. P. Sociomicrobiology: the connections between quorum sensing and biofilms. Trends Microbiol 13, 27-33, doi:10.1016/j.tim.2004.11.007 (2005). Microbiol 6, 199-210, doi:10.1038/nrmicro1838 (2008).

23 Moreno-Gamez, S. et al. Quorum sensing integrates environmental cues, cell density and cell history to control bacterial competence. Nat Commun 8, 854, doi:10.1038/s41467-

24 Singh, P. K. et al. Vibrio cholerae Combines Individual and Collective Sensing to Trigger Biofilm Dispersal. Curr Biol 27, 3359-3366 e3357, doi:10.1016/j.cub.2017.09.041 (2017).

25 Shadel, G. S. \& Baldwin, T. O. Positive autoregulation of the Vibrio fischeri luxR gene. LuxR and autoinducer activate cAMP-catabolite gene activator protein complexindependent and -dependent luxR transcription. J Biol Chem 267, 7696-7702 (1992).

26 Miyashiro, T. \& Ruby, E. G. Shedding light on bioluminescence regulation in Vibrio fischeri. Mol Microbiol 84, 795-806, doi:10.1111/j.1365-2958.2012.08065.x (2012).

27 Shadel, G. S. \& Baldwin, T. O. The Vibrio fischeri LuxR protein is capable of bidirectional stimulation of transcription and both positive and negative regulation of the luxR gene. J Bacteriol 173, 568-574 (1991).

28 Stevens, A. M., Dolan, K. M. \& Greenberg, E. P. Synergistic binding of the Vibrio fischeri LuxR transcriptional activator domain and RNA polymerase to the lux promoter region. Proc Natl Acad Sci US A 91, 12619-12623 (1994). Haseltine, E. L. \& Arnold, F. H. Implications of rewiring bacterial quor
Environ Microbiol 74, 437-445, doi:10.1128/AEM.01688-07 (2008).

30 Williams, J. W., Cui, X., Levchenko, A. \& Stevens, A. M. Robust and sensitive control of a quorum-sensing circuit by two interlocked feedback loops. Mol Syst Biol 4, 234, doi:10.1038/msb.2008.70 (2008). most out of nutrients. Nat Rev Microbiol 6, 613-624, doi:10.1038/nrmicro1932 (2008). Dunlap, P. V. \& Greenberg, E. P. Control of Vibrio fischeri lux gene transcription by a cyclic AMP receptor protein-luxR protein regulatory circuit. J Bacteriol 170, 4040-4046 (1988).

33 Goryachev, A. B., Toh, D. J. \& Lee, T. Systems analysis of a quorum sensing network: design constraints imposed by the functional requirements, network topology and kinetic constants. Biosystems 83, 178-187, doi:10.1016/j.biosystems.2005.04.006 (2006).

34 Melke, P., Sahlin, P., Levchenko, A. \& Jonsson, H. A cell-based model for quorum sensing in heterogeneous bacterial colonies. PLoS Comput Biol 6, e1000819, doi:10.1371/journal.pcbi.1000819 (2010).

35 Cheong, R., Rhee, A., Wang, C. J., Nemenman, I. \& Levchenko, A. Information transduction capacity of noisy biochemical signaling networks. Science 334, 354-358, doi:10.1126/science.1204553 (2011).

36 Rhee, A., Cheong, R. \& Levchenko, A. The application of information theory to biochemical signaling systems. Phys Biol 9, 045011, doi:10.1088/1478-3975/9/4/045011 (2012). 
82637 Bauer, M., Knebel, J., Lechner, M., Pickl, P. \& Frey, E. Ecological feedback in quorumsensing microbial populations can induce heterogeneous production of autoinducers. Elife 6, doi:10.7554/eLife.25773 (2017).

43 Ribbe, J. \& Maier, B. Density-Dependent Differentiation of Bacteria in Spatially

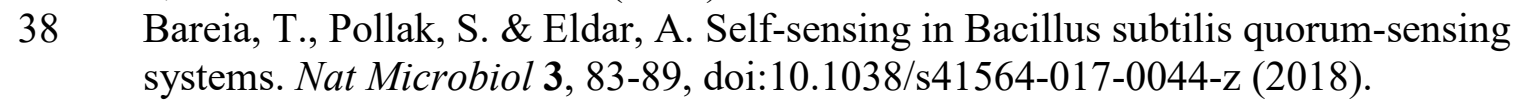

39 Beaumont, H. J., Gallie, J., Kost, C., Ferguson, G. C. \& Rainey, P. B. Experimental evolution of bet hedging. Nature 462, 90-93, doi:10.1038/nature08504 (2009).

40 Solopova, A. et al. Bet-hedging during bacterial diauxic shift. Proc Natl Acad Sci U S A 111, 7427-7432, doi:10.1073/pnas.1320063111 (2014).

41 You, C. et al. Coordination of bacterial proteome with metabolism by cyclic AMP signalling. Nature 500, 301-306, doi:10.1038/nature12446 (2013).

42 Giordano, N., Mairet, F., Gouze, J. L., Geiselmann, J. \& de Jong, H. Dynamical Allocation of Cellular Resources as an Optimal Control Problem: Novel Insights into Microbial Growth Strategies. PLoS Comput Biol 12, e1004802, doi:10.1371/journal.pcbi.1004802 (2016).

\section{Acknowledgements}

856 We thank A. Stevens for the generous gifts of the cell strain used in this study. We are grateful to

857 everyone in the Levchenko group for the helpful discussions.

\section{$\underline{\text { Author Contributions }}$}

859 E.K.C., A.G. and A.L. designed the device and E.K.C. fabricated the device. E.K.C. performed

860 experiments and simulations, and analyzed data. E.K.C. and A.L. wrote the paper.

\section{Competing Interests}

862 The authors declare no competing interests. 

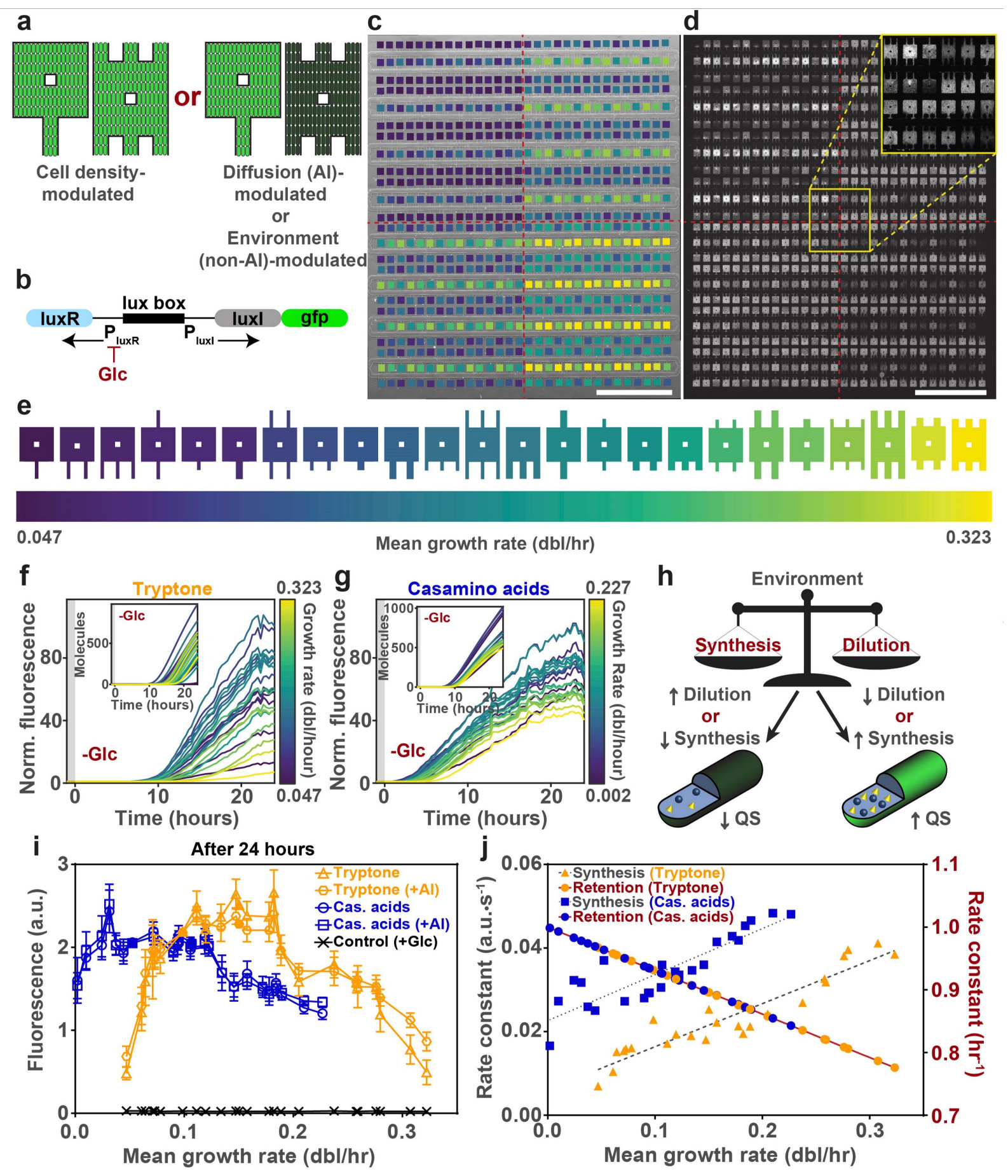

864 Figure 1 QS is defined by a balance between nutrient-defined protein synthesis and dilution

865 due to cell proliferation. a, QS is traditionally interpreted as either a mechanism for cell

866 number/density sensing or diffusive environment sensing, which would lead to different response

867 outcomes in certain environments. b, Diagram of QS circuit used in the study. $\mathbf{c}$, Phase contrast 
868 micrograph of microfluidic device chamber array overlaid with color-coding corresponding to

869 the 24 unique chamber configurations. Scale bar, $1 \mathrm{~mm}$. d, Fluorescence micrograph of

870 microfluidic device chamber array taken 24 hours after auto-induced QS response in tryptone

871 medium at $25^{\circ} \mathrm{C}$. Inset contain magnified view of the region indicated, which contains all 24

872 chamber configurations. Scale bar, $1 \mathrm{~mm}$. e, Color-coding of chamber configurations based on

873 mean growth rate in tryptone medium. f, $\mathbf{g}$, Auto-induction response dynamics over 24 hours in

874 tryptone (f) and casamino acids (g) media. (mean, $\mathrm{n}=6$, from 3 independent experiments). Insets

875 denote simulated dynamics obtained by varying only synthesis and dilution with parameters from

876 (j). h, Proposed regulatory mechanism of QS response based on the balance of synthesis and

877 dilution of QS machinery proteins. i, Distributions of QS response magnitudes after 24 hours in

878 various nutrient and induction conditions. Filled shapes indicate the 2 chamber types excluded

879 from this analysis due to partial formation of cell bilayer (Supp. Info.) $(n=105$ or $n=60$, for

880 single- or double-sided chambers, respectively, from 3 independent experiments, mean \pm SD). j,

881 Measured synthesis and dilution (growth) rate constants from tryptone and casamino acids media

882 conditions. 


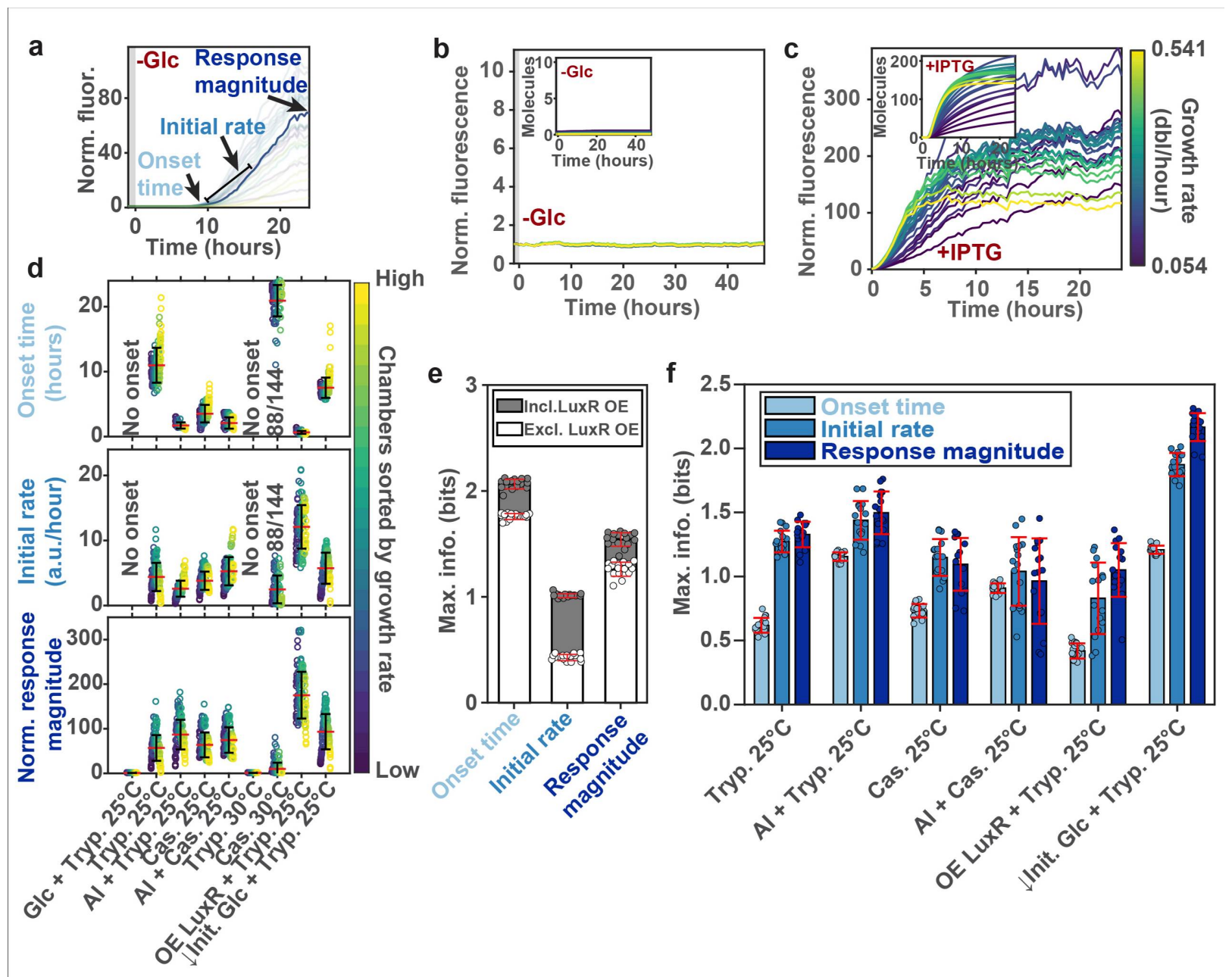

Figure 2 Information about distinct environmental conditions is reflected in QS response and $24^{\text {th }}$ hour response magnitude. b,c, Response dynamics within, (b) tryptone medium at $30^{\circ} \mathrm{C}$, and (c) tryptone medium with $1 \mathrm{mM}$ IPTG (for LuxR overexpression) at $30^{\circ} \mathrm{C}$ (mean, $\mathrm{n}=6$,

889 from 3 independent experiments). Insets denote simulated dynamics. d, Summary of onset times, 890 initial rates, and response magnitudes from various conditions $(n=144$, from 3 independent

891 experiments). e, Comparison of information about global environmental conditions discerned by

892 each component of QS dynamics assuming all chamber configurations are equivalent. f,

893 Comparison of information about the local differential coupling environment discerned by each 894 component of QS dynamics for various global environmental conditions. (mean $\pm \mathrm{SD}$ ) 


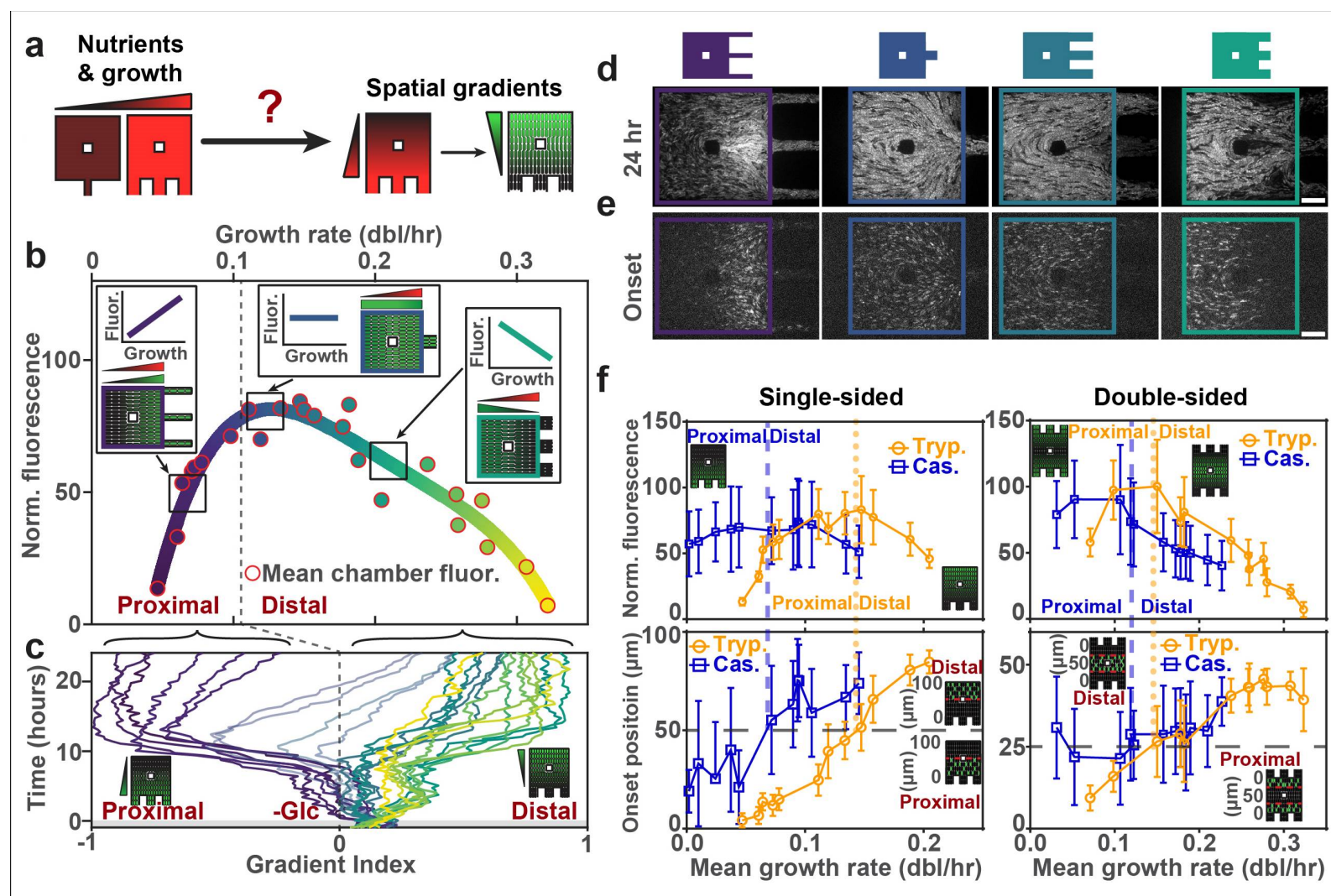

Figure 3 Spatial properties of environmentally regulated QS response dependence. a,

897 Spatial dependence could arise from nutrient and growth gradients within the chamber. b, The

898 proposed relationship between spatial response gradients and the biphasic response magnitude

899 distribution. c, Quantification of auto-induction spatial response gradient dynamics over 24 hours

900 in tryptone medium at $25^{\circ} \mathrm{C}$. Positive gradient index indicates higher response in the regions

901 distal from the coupling channels, whereas negative gradient index indicates higher response in

902 the regions proximal from the coupling channels. (mean, $n=6$, from 3 independent

903 experiments). d,e, Fluorescence micrographs of chamber configurations corresponding to those

904 illustrated in (b), at $24^{\text {th }}$ hour (d) and at onset (e). Scale bar, $20 \mu \mathrm{m}$. f, Spatial localization of

905 auto-induced QS response onset in single- and double-sided chambers in tryptone and casamino

906 acid media at $25^{\circ} \mathrm{C}$, in relation to response magnitude distributions after 24 hours in the same

907 conditions $(n=43-64$, from 3 independent experiments) (mean \pm SD). 


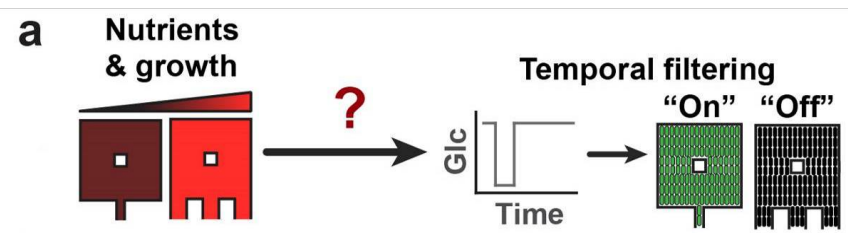

b

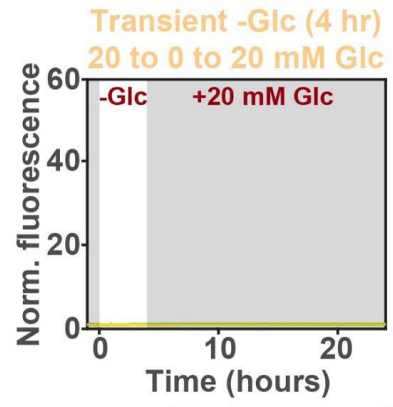

C

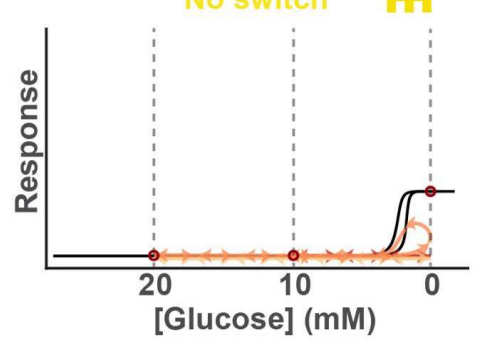

d

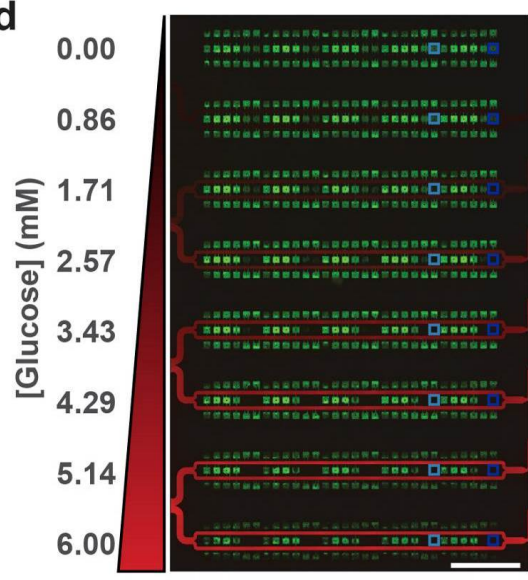

Condition 2:

Chamber 2.

e

f
Transient -GIc (16 hr)

20 to 0 to $20 \mathrm{mM}$ Glc

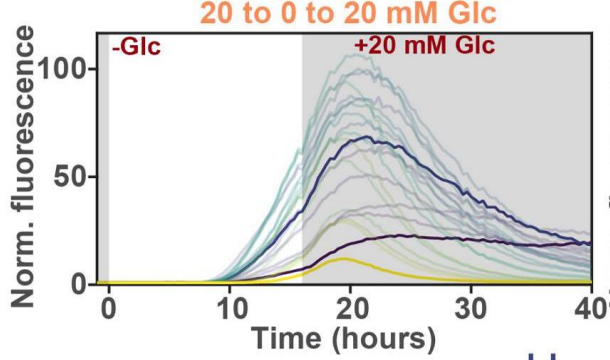

Switch in condition 3
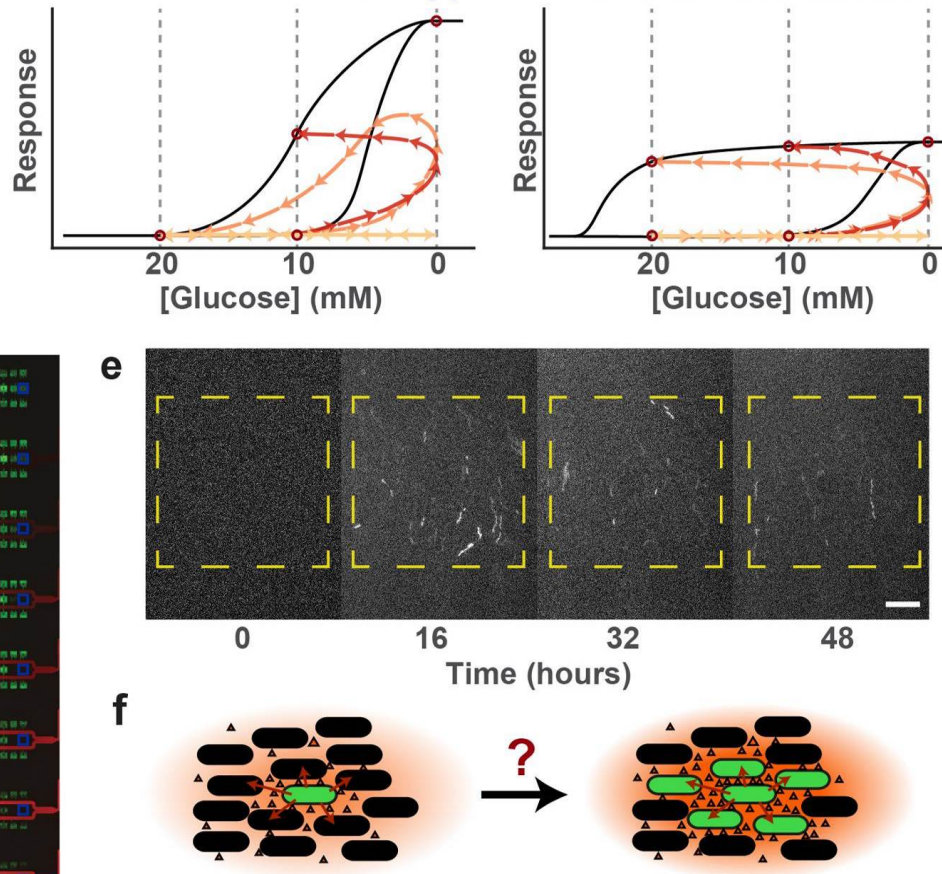

Induced subpopulation
Condition 3:

Transient -Glc (4 hr, $\downarrow$ [GIc])

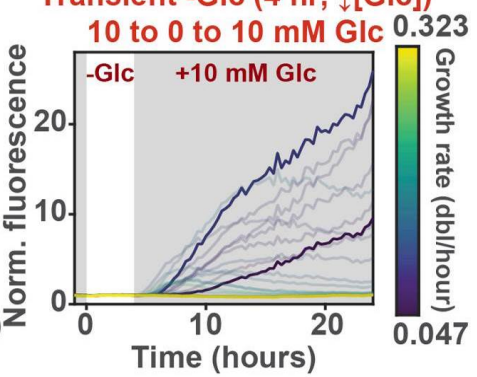

Chamber 3:

Switch in conditions 28.3

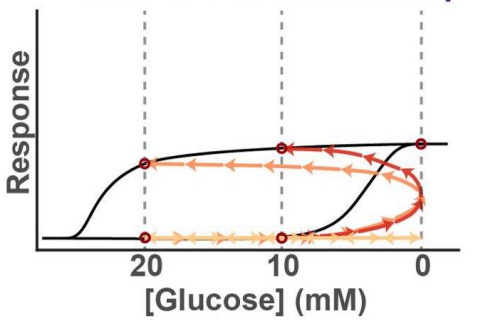

[Glucose] (mM)

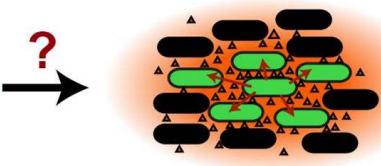

$\downarrow$ Temporal threshold

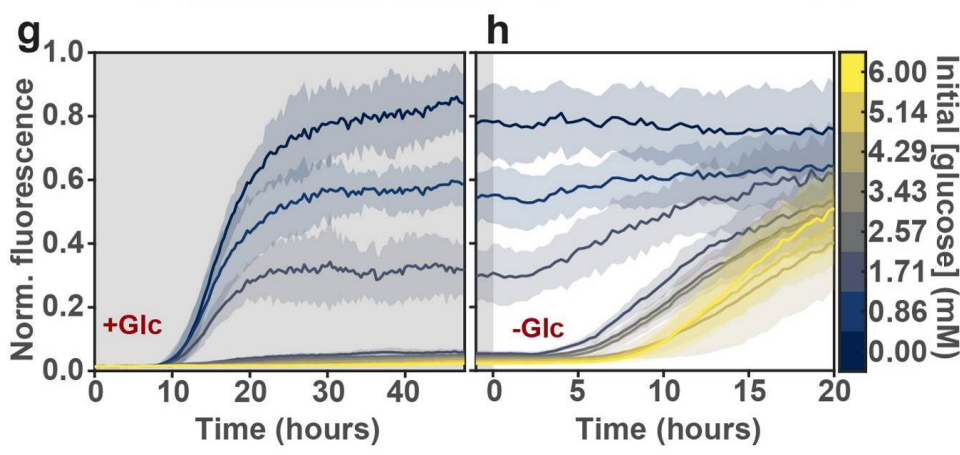

i Initial states

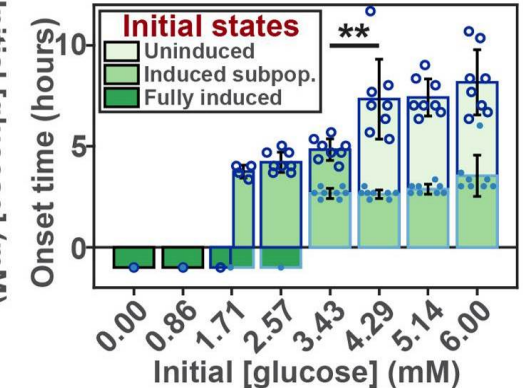


909 Figure 4 Sensitivity to environmental change as encoded in the form of a temporal

910 threshold can be enhanced with cell signaling. a, QS onset can be controlled by a condition-

911 dependent temporal threshold during transient stimulation. b, Response dynamics of 4 hour

912 transient glucose $(20 \mathrm{mM})$ removal (condition 1), 16 hour transient glucose $(20 \mathrm{mM})$ removal

913 (condition 2), and 4 hour transient glucose (10 mM) removal (condition 3). All conditions were

914 in tryptone medium at $25^{\circ} \mathrm{C}$. (mean, $\mathrm{n}=6$, from 3 independent experiments). 3 sample chambers

915 in each condition have been highlighted. c, Theoretical hysteretic diagrams of three sample

916 chambers within the three conditions in b. d, Fluorescence micrograph of gradient-generating

917 device, with chambers of interest indicated in blue boxes and the gradient visualized in red. Scale

918 bar, $1 \mathrm{~mm}$. e, Filmstrip of a representative chamber exposed to $2.57 \mathrm{mM}$ glucose demonstrating

919 maintenance of a stably induced subpopulation over $\sim 40$ hours. Scale bar, $20 \mu \mathrm{m}$. f, Proposed

920 effects of an induced subpopulation. $\mathbf{g}$, Response dynamics from chambers exposed to $0-6 \mathrm{mM}$

921 linearly graded glucose concentrations over 48 hours $(n=8) . \mathbf{h}$, Response dynamics over 24

922 hours after glucose removal $(n=8)$. i, Onset time of chambers exposed to different initial

923 concentrations of glucose. ( $\mathrm{n}=8, * * P<0.01$, two tailed Student's $t$-test). (mean $\pm \mathrm{SD})$ 